\title{
Study on the Multitarget Mechanism and Active Compounds of Essential Oil from Artemisia argyi Treating Pressure Injuries Based on Network Pharmacology
}

\author{
Shu-ting Lu $\mathbb{D},{ }^{1}$ Lu-lu Tang $\mathbb{D}^{1},{ }^{1}$ Ling-han Zhou $\mathbb{D}^{\mathbb{D}},{ }^{1}$ Ying-tao Lai $\mathbb{D}^{2},{ }^{2}$ Lan-xing Liu $\mathbb{D},{ }^{1}$ \\ and Yifan Duan (iD) ${ }^{1}$ \\ ${ }^{1}$ The First College of Clinical Medicine, Guangzhou University of Chinese Medicine, Guangzhou, China \\ ${ }^{2}$ Department of Anesthesiology, The First Affiliated Hospital, Guangzhou University of Chinese Medicine, Guangzhou, China \\ Correspondence should be addressed to Ying-tao Lai; 1131251355@qq.com
}

Received 30 September 2021; Revised 30 November 2021; Accepted 23 December 2021; Published 19 January 2022

Academic Editor: Mozaniel Oliveira

Copyright (c) 2022 Shu-ting Lu et al. This is an open access article distributed under the Creative Commons Attribution License, which permits unrestricted use, distribution, and reproduction in any medium, provided the original work is properly cited.

\begin{abstract}
In order to comprehensively explore multitarget mechanism and key active compounds of Artemisia argyi essential oil (AAEO) in the treatment of pressure injuries (PIs), we analyzed the biological functions and pathways involved in the intersection targets of AAEO and PIs based on network pharmacology, and the affinity of AAEO active compounds and core targets was verified by molecular docking finally. In our study, we first screened 54 effective components according to the relative content and biological activity. In total, 103 targets related to active compounds of AAEO and 2760 targets associated with PIs were obtained, respectively, and 50 key targets were overlapped by Venny 2.1.0. The construction of key targets-compounds network was achieved by the STRING database and Cytoscape 3.7.2 software. GO analysis from Matespace shows that GO results are mainly enriched in biological processes, including adrenergic receptor activity, neurotransmitter clearance, and neurotransmitter metabolic process. KEGG analysis by the David and Kobas website shows that the key targets can achieve the treatment on PIs through a pathway in cancer, PI3K-Akt signaling pathway, human immunodeficiency virus 1 infection, MAPK signaling pathway, Wnt signaling pathway, etc. In addition, molecular docking results from the CB-Dock server indicated that active compounds of AAEO had good activity docking with the first 10 key targets. In conclusion, the potential targets and regulatory molecular mechanisms of AAEO in the treatment of PIs were analyzed by network pharmacology and molecular docking. AAEO can cure PIs through the synergistic effect of multicomponent, multitarget, and multipathway, providing a theoretical basis and new direction for further study.
\end{abstract}

\section{Introduction}

Pressure injuries (PIs), also named pressure ulcers, refer to localized injuries occurring in the skin and/or potential subcutaneous soft tissue, usually occurring in bone bulges or in contact with medical facilities [1]. PIs have the characteristics of refractory, high incidence, and high treatment cost $[2,3]$. Once infected, it is easy to cause sepsis and death [4]. At present, the treatment of PIs mainly includes drug therapy [5], dressing therapy [6], stem cell factor therapy [7], and negative pressure wound therapy [8]. There are no effective measures yet; expert consensus believes that prevention and early treatment are crucial [9].
The Artemisia argyi (AA), which is widely distributed in China and other Asian countries, has been used as traditional medicine or food supplement for hundreds of years [10]. AA is the dried leaf of Artemisia argyi (Levl.) et Van., the herb with a spicy, bitter flavor and warm properties, enters into the channels of liver and kidney, and functions on resolving blood stasis, dispersing cold and relieving pain $[11,12]$. AA is rich in volatile essential oils (AAEO), such as eucalyptol, camphor, and borneol, with extensive pharmacological effects of antioxidative stress [13], resisting pathogens [14], suppressing inflammatory responses [15], and activating immunomodulatory responses [16]. 
AA often treats diseases in the form of moxibustion; moxibustion is a critical intervention in traditional Chinese medicine (TCM). Artemisia argyi is usually the main raw material [17]. Although the mechanism of moxibustion is uncertain, the thermal effect and moxa smoke may play a synergistic role in the treatment of diseases [18, 19]. The fumigation and heating effects produced by moxibustion have played a certain role in promoting the wound healing of PIs, and the pharmacological effects of moxa smoke need to be paid special attention. Nevertheless, we found that moxa smoke and AAEO have $80 \%$ of the same compounds by searching the relevant literature. Also, in view of the increasing emphasis on the toxicity of moxa smoke to cardiovascular and respiratory systems, AAEO is safer.

In view of the complex chemical compounds of AAEO, the chemical components and the corresponding mechanism of action that play the efficacy after entering the human body include a lot of unknown information. Therefore, it is necessary to comprehensively explore the mechanism of AAEO in the treatment of PIs.

Network pharmacology is a new discipline emerging in recent years that combines the overall network analysis and pharmacological effects [20]. With the development of bioinformatics and chemical informatics, network pharmacology has become a new method to study the mechanism of traditional drugs and discover potential bioactive components effectively and systematically [21]. Network pharmacology explores the relationship between drugs and diseases from a holistic perspective and, through a large number of databases screening drug treatment of diseases related targets and pathways, is widely used in TCM-related fields, providing new ideas for the study of complex Chinese medicine system [20, 22, 23]. Molecular docking, as a new technology for drug molecular screening, utilizes one-to-one pairs of ligands and receptors according to the "lock-key principle," the computer-aided high-throughput screening of drug molecules was realized by studying the geometric matching and energy matching between protein macromolecular receptors and small drug molecules, and the mechanism of drug molecules was further predicted to improve the scientificity, accuracy, sensitivity, and predictability of drug molecule screening [24].

For all we know, our study is first time applied network pharmacology methods to explore the biological effect of active compounds in AAEO and the multitarget mechanism of active compounds in the treatment of PIs. In our study, TNF, PTGS2, IL6, IL1 $\beta$, NR3C1, CASP3, TP53, PGR, REN, and NOS 2 could be the potential receptor targets, involving many inflammatory proteins. The top three molecular docking points are PTGS2 (prostaglandin-endoperoxide synthase 2), TP53 (tumor protein p53), and PGR (progesterone receptor). PTGS2, also known as COX-2, as an important inflammatory mediator, exists in the early stage of inflammation to the whole process of inflammation formation [25]. It is upregulated when stimulated by various stimuli and participates in various pathological processes, closely related to inflammation, tumor occurrence, and development $[26,27]$. TP53 and PGR are tumor suppressor proteins, being a biomarker and prognostic predictor of cancers usually [28-31]. Recent studies have shown that TP53 plays an important role in regulating signaling pathways to maintain the health and function of skeletal muscle cells. It can improve cell survival rate by participating in the activation to increase the repair time of cells and prevent abnormal cell proliferation through the initiation of DNA fragmentation-induced apoptosis to promote the increase of cell stress level [32].

\section{Methods}

2.1. Active Compounds of AAEO Database Building and Screening. Over 200 components of AAEO can be detected by current technology, but more than 90 of them are common active, so we use 94 components as active compounds [33, 34]. Fifty-four compounds were screened by criteria. Finally, the inclusion criteria were as follows: the compounds with relative content $>0.1 \%$ from works of literature of GS-MC quantitative analysis (hydrodistillation) of AAEO in recent years $[14,35,36]$, compounds included in TCMSP [37] (https://tcmspw.com) and PubChem database [38] (https://pubchem.ncbi.nlm.nih.gov/), and compounds with relevant targets.

2.2. Targets Fishing. The targets information identifying 54 potential compounds were attained on TCMSP and were reconfirmed by DrugBank [39] (https://www.drugbank.ca) and Pharmmapper [40] (https://www.lilab-ecust.cn/ pharmmapper/). Next, the targets were entered into UniProt (https://www.uniprot.org/); the species selected was "Homo sapiens"; transformed gene symbols were obtained finally.

GeneCard (https://www.genecards.org/), OMIM (https://omim.org/) and DrugBank (https://go.drugbank. $\mathrm{com} /$ ) database were used to screen relative targets of PIs. "Pressure Ulcers," "Bedsore," "Pressure Sore," and "pressure injury" were keywords to search targets related to PIs. The obtained targets were integrated and eliminated duplication. Finally, the intersection targets were obtained on Venny 2.1.0 (https://bioinfogp.cnb.csic.es/tools/venny/). At last, 50 overlapping targets were obtained.

2.3. PPI Analysis and Compounds-Targets Network Construction. PPI analysis of the overlapping targets was carried out in the STRING 11.0 (https://www.string-db.org/). Protein with disconnected other protein and a combined score $<0.4$ was removed [41]. The information of the PPI network was visualized by Cytoscape 3.7.2 software [42]; then, core network calculations were performed by the Cytoscape plug-in module, MCODE, the degree of freedom threshold was set as 100 , the node scoring threshold was 0.2 , the $K$ value was 2 , and the maximum depth was 100 [43].

2.4. Gene Ontology (GO) Analysis. The overlapping targets were imported into Matescape [44] (https://metascape.org/ gp/index.html) to carry out GO analysis. The specific steps were as follows: input the gene ID, the parameter selected 
was "Homo sapiens," click "custom analysis," and click GO Molecular Functions, GO Biological Processes, and GO Cellular Components in turn for analysis [44]. Finally, Bioinformatics (https://www.bioinformatics.com.cn/) was used to acquire the visualization of the results.

2.5. Kyoto Encyclopedia of Genes and Genomes (KEGG) Pathways Analysis. 50 overlapping targets were converted from gene symbol to ENTRZ_GENE ID in David Database (https://david.ncifcrf.gov/tools.jsp), and the ENTRZ_GENE ID was input into Kobas (https://kobas.cbi.pku.edu.cn/) for KEGG pathways analysis $[45,46]$. KEGG pathways with $P$ values $<0.01$ were selected [47].

2.6. Molecular Docking. In silico methods are alternatives to experimental approaches to screen for potential bioactivity of compounds of essential oil compounds; for example, docking evaluated in silico the ability of EOs to interact with molecular targets with advantages of being less time-consuming and cheap. We selected the top 10 core targets and got the ligand with relative content of the first 7 for molecular docking; the PDB formats of proteins were obtained from the protein database (https://www.rcsb.org) and ligand files in mol2 formats from PubChem (https://pubchem.ncbi. nlm.nih.gov/) [48]; both of them were used in the same way they were obtained from the databases. Molecular docking was carried out in CB-Dock (https://cao.labshare.cn/cbdock/). CB-Dock server is a user-friendly blind docking network server developed by Dr. Liu's research team. It uses a novel curvature-based cavity detection approach, and Autodock Vina, the popular docking program, is used for docking [49]. The success rate of this tool was more than $70 \%$, which outperformed the state-of-the-art blind docking tools. The downloaded formats files were input into CBDock; the style and color of ligand and receptor were set the same as those of Dr. Tao [50]. The RMSD between each pair of the two structures must be less than 2 angstroms [51].

\section{Results}

3.1. Compounds of AAEO and Targets Related to Active Compounds. A total of 54 active compounds that met the criteria were finally collected. The basic information of 54 obtained compounds is shown in Table 1.

\subsection{Targets' Intersection and PPI Network Construction.} 103 AAEO compound-related targets were retrieved from TCMSP and converted into official gene symbols according to the UniProt database. Moreover, 2760 PIs targets were searched by GeneCard, OMIM, and DrugBank databases. Finally, 50 targets were obtained by intersecting two parts of targets (Figure 1); the PPIs of 50 overlapping targets are shown in Figure 2.

3.3. Active Compounds and Overlapping Targets Network Construction. Compounds-overlapping targets network involved 104 nodes and 441 edges. The results reflect the complex mechanism of multicomponent and multitarget treatment of diseases. Moreover, a core network was calculated by MCODE with 15 targets (Figure 3).

3.4. GO Analysis of Targets' Intersection. GO analysis was mainly focused on the biological process, with a total of 3269 enrichment results, involving adrenergic receptor activity, nuclear receptor activity, and aspartic-type endopeptidase activity. The top $10 \mathrm{GO}$ functional annotations of BP, CC, and MF are shown in Figure 4.

The top 10 GO functional annotations of BP, CC, and $\mathrm{MF}$ are represented by green for biological process, orange for cellular component, light purple for molecular function, respectively.

3.5. KEGG Pathways of Targets' Intersection. KEGG enrichment results were involved in 128 pathways, including pathway in cancer, PI3K-Akt signaling pathway, human immunodeficiency virus 1 infection, MAPK signaling pathway, and Wnt signaling pathway. The top 20 pathways were selected by cluster analysis and $P$-value (Figure 5).

Each bubble represents an enriched function, and the size of the bubble is from small to large. The bubble is colored according to its $-\log (P$ value); when the color is redder, $P$ value is smaller.

3.6. Compound-Target Docking. The 10 key targets, TNF, PTGS2, IL6, IL1 $\beta$, NR3C1, CASP3, TP53, PGR, REN, and NOS2, were docked with top 7 compounds: $\beta$-caryophyllene (A27),1,8-cineole (A1), terpinen-4-ol (A51), neointermedeol (A4), $\alpha$-thujone (A11), borneol (A6), and camphor (A3). Generally, the Vina score is negative; the lower the score, the better the binding activity between ligand and protein. There will be top five Vina scores and docking cavity sizes from obtained results, which were first selected as representation [50]. The results indicated that the top 7 active compounds of AAEO had a good affinity to key targets and the RMSD of each docking target and compound was less than 2 angstroms (Tables 2 and 3). The top 3 compounds (A4neointermedeol, A27- $\beta$-caryophyllene, and A3-camphor) and proteins (PTGS2, PGR, and TP53) with better binding affinities are shown in Figures 6-8.

\section{Discussion}

Artemisia argyi, a dried leaf of Ai Ye with multiple biological activities, is widely used to treat inflammatory diseases such as eczema, dermatitis, arthritis, allergic asthma, and colitis [52]. The pharmacological mechanisms of AAEO associated with PIS are uncertain. Our study was first used network pharmacology to discover the potential targets and regulatory molecular mechanism of AAEO on PIs treatment. As a result, we identified 54 compounds as the main active components, obtained 50 key targets, including pathway in cancer, PI3K-Akt signaling pathway, human immunodeficiency virus 1 infection, MAPK signaling pathway, and Wnt 
TABLE 1: The basic information of potential compounds of AAEO.

\begin{tabular}{|c|c|c|c|c|c|}
\hline No. & Molecule name & CAS & Molecular formula & Relative content (\%) & References \\
\hline $\mathrm{A} 1$ & 1,8-Cineole & $470-82-6$ & $\mathrm{C}_{10} \mathrm{H}_{18} \mathrm{O}$ & 20.91 & Guan et al. [14] \\
\hline $\mathrm{A} 2$ & Caryophyllene & $87-44-5$ & $\mathrm{C}_{15} \mathrm{H}_{24}$ & 7.50 & Guan et al. [14] \\
\hline $\mathrm{A} 3$ & (-)-Camphor & $76-22-2$ & $\mathrm{C}_{10} \mathrm{H}_{16} \mathrm{O}$ & 5.57 & Guan et al. [14] \\
\hline A4 & Neointermedeol & $5945-72-2$ & $\mathrm{C}_{15} \mathrm{H}_{26} \mathrm{O}$ & 9.65 & Guan et al. [14] \\
\hline A5 & Caryophyllene oxide & $1139-30-6$ & $\mathrm{C}_{15} \mathrm{H}_{24} \mathrm{O}$ & 8.71 & Guan et al. [14] \\
\hline A6 & (-)-Borneol & $464-45-9$ & $\mathrm{C}_{10} \mathrm{H}_{18} \mathrm{O}$ & 16.35 & Guan et al. [14] \\
\hline A7 & D-Carvone & $5948 / 4 / 9$ & $\mathrm{C}_{10} \mathrm{H}_{16} \mathrm{O}$ & 0.25 & Guan et al. [14] \\
\hline A8 & Bornyl acetate & $76-49-3$ & $\mathrm{C}_{12} \mathrm{H}_{20} \mathrm{O}_{2}$ & 0.24 & Guan et al. [14] \\
\hline A9 & 4-Terpineol & $562-74-3$ & $\mathrm{C}_{10} \mathrm{H}_{18} \mathrm{O}$ & 5.47 & Guan et al. [14] \\
\hline A 10 & Sabinene & $10408-16-9$ & $\mathrm{C}_{10} \mathrm{H}_{16}$ & 3.36 & Guan et al. [14] \\
\hline A11 & $\alpha$-Thujone & $546-80-5$ & $\mathrm{C}_{10} \mathrm{H}_{16} \mathrm{O}$ & 14.55 & Guan et al. [14] \\
\hline A12 & $\alpha$-Humulene & $6753-98-6$ & $\mathrm{C}_{15} \mathrm{H}_{24}$ & 2.24 & Guan et al. [14] \\
\hline A13 & Eugenol & $97-53-0$ & $\mathrm{C}_{10} \mathrm{H}_{12} \mathrm{O}_{2}$ & 0.56 & Gu et al. [36] \\
\hline A14 & cis-Carveol & $1197-06-4$ & $\mathrm{C}_{10} \mathrm{H}_{16} \mathrm{O}$ & 1.40 & Guan et al. [14] \\
\hline A15 & Germacrene D & $23986-74-5$ & $\mathrm{C}_{15} \mathrm{H}_{24}$ & 0.55 & Guan et al. [14] \\
\hline A16 & Terpinolene & $586-62-9$ & $\mathrm{C}_{10} \mathrm{H}_{16}$ & 0.15 & Guan et al. [14] \\
\hline A17 & Cymene & $527-84-4$ & $\mathrm{C}_{10} \mathrm{H}_{14}$ & 0.32 & Guan et al. [14] \\
\hline A18 & $\alpha$-Terpineol & $10482-56-1$ & $\mathrm{C}_{10} \mathrm{H}_{18} \mathrm{O}$ & 3.62 & Guan et al. [14] \\
\hline A19 & cis-Carveol & $1197-06-4$ & $\mathrm{C}_{10} \mathrm{H}_{16} \mathrm{O}$ & 1.40 & Guan et al. [14] \\
\hline A 20 & Espatulenol & $6750-60-3$ & $\mathrm{C}_{15} \mathrm{H}_{24} \mathrm{O}$ & 1.51 & Guan et al. [14] \\
\hline $\mathrm{A} 21$ & $\gamma$-Elemene & $515-13-9$ & $\mathrm{C}_{15} \mathrm{H}_{24}$ & 0.12 & Gu et al. [36] \\
\hline A 22 & $\alpha$-Pinene & $2437-95-8$ & $\mathrm{C}_{10} \mathrm{H}_{16}$ & 3.84 & Dai et al. [35] \\
\hline $\mathrm{A} 23$ & Piperitone & $89-81-6$ & $\mathrm{C}_{10} \mathrm{H}_{16} \mathrm{O}$ & 0.42 & Guan et al. [14] \\
\hline A24 & (-)-Camphene & $5794 / 3 / 6$ & $\mathrm{C}_{10} \mathrm{H}_{16}$ & 1.83 & Dai et al. [35] \\
\hline A 25 & Isoborneol & $124-76-5$ & $\mathrm{C}_{10} \mathrm{H}_{18} \mathrm{O}$ & 0.63 & Dai et al. [35] \\
\hline A26 & cis- $\beta$-Farnesene & $18794-84-8$ & $\mathrm{C}_{15} \mathrm{H}_{24}$ & 0.11 & Dai et al. [35] \\
\hline $\mathrm{A} 27$ & $\beta$-Caryophyllene & $87-44-5$ & $\mathrm{C}_{15} \mathrm{H}_{24}$ & 13.64 & Guan et al. [14] \\
\hline A28 & $\gamma$-Terpinene & $99-85-4$ & $\mathrm{C}_{10} \mathrm{H}_{16}$ & 0.24 & Guan et al. [14] \\
\hline A29 & Spathulenol & $4221-98-1$ & $\mathrm{C}_{15} \mathrm{H}_{24} \mathrm{O}$ & 0.82 & Dai et al. [35] \\
\hline A30 & Diisooctyl phthalate & $27554-26-3$ & $\mathrm{C}_{24} \mathrm{H}_{38} \mathrm{O}_{4}$ & 0.14 & Dai et al. [35] \\
\hline A31 & $\beta$-Pinene & $127-91-3$ & $\mathrm{C}_{10} \mathrm{H}_{16}$ & 3.05 & Dai et al. [35] \\
\hline A32 & Hexahydrofarnesyl acetone & $502-69-2$ & $\mathrm{C}_{18} \mathrm{H}_{36} \mathrm{O}$ & 0.77 & Dai et al. [35] \\
\hline A33 & Tricyclene & $508-32-7$ & $\mathrm{C}_{10} \mathrm{H}_{16}$ & 0.12 & Gu et al. [36] \\
\hline A 34 & Terpinene & $99-86-5$ & $\mathrm{C}_{10} \mathrm{H}_{16}$ & 2.26 & Gu et al. [36] \\
\hline A35 & Dihydroactinidiolide & $15356-74-8$ & $\mathrm{C}_{11} \mathrm{H}_{16} \mathrm{O}_{2}$ & 0.21 & Dai et al. [35] \\
\hline A 36 & Cyclohexadiene & $4221-98-1$ & $\mathrm{C}_{10} \mathrm{H}_{16}$ & 0.77 & Dai et al. [35] \\
\hline A37 & n-Hexadecanoic acid & $57-10-3$ & $\mathrm{C}_{16} \mathrm{H}_{32} \mathrm{O}_{2}$ & 0.22 & Dai et al. [35] \\
\hline A 38 & Terpinyl acetate & 58206-95-4 & $\mathrm{C}_{12} \mathrm{H}_{20} \mathrm{O}_{2}$ & 0.27 & Dai et al. [35] \\
\hline A39 & Diisobutyl phthalate & 84-69-5 & $\mathrm{C}_{16} \mathrm{H}_{22} \mathrm{O}_{4}$ & 0.14 & Dai et al. [35] \\
\hline $\mathrm{A} 40$ & Myrtenol & $19894-97-4$ & $\mathrm{C}_{10} \mathrm{H}_{16} \mathrm{O}$ & 0.77 & Dai et al. [35] \\
\hline A41 & Carvacrol & 499-75-2 & $\mathrm{C}_{10} \mathrm{H}_{14} \mathrm{O}$ & 0.55 & Dai et al. [35] \\
\hline $\mathrm{A} 42$ & Curcumene & $4176-17-4$ & $\mathrm{C}_{15} \mathrm{H}_{22}$ & 1.06 & Dai et al. [35] \\
\hline A 43 & trans-Carveol & $2102-58-1$ & $\mathrm{C}_{10} \mathrm{H}_{16} \mathrm{O}$ & 1.17 & Dai et al. [35] \\
\hline A 44 & $(+)$-Limonene & $5989-27-5$ & $\mathrm{C}_{10} \mathrm{H}_{16}$ & 0.39 & Dai et al. [35] \\
\hline A 45 & L-Carvone & $6485-40-1$ & $\mathrm{C}_{10} \mathrm{H}_{14} \mathrm{O}$ & 0.11 & Dai et al. [35] \\
\hline A 46 & cis- $\beta$-Terpineol & $7299-40-3$ & $\mathrm{C}_{10} \mathrm{H}_{18} \mathrm{O}$ & 6.61 & Dai et al. [35] \\
\hline A 47 & cis-Piperitol & $16721-38-3$ & $\mathrm{C}_{10} \mathrm{H}_{18} \mathrm{O}$ & 3.66 & Dai et al. [35] \\
\hline A 48 & Nerolidol & $7212-44-4$ & $\mathrm{C}_{15} \mathrm{H}_{26} \mathrm{O}$ & 0.59 & Dai et al. [35] \\
\hline A 49 & cis-Jasmon & $488-10-8$ & $\mathrm{C}_{11} \mathrm{H}_{16} \mathrm{O}$ & 0.42 & Dai et al. [35] \\
\hline A 50 & $\alpha$-Caryophyllene & $6753-98-6$ & $\mathrm{C}_{15} \mathrm{H}_{24}$ & 0.37 & Dai et al. [35] \\
\hline A51 & Terpinen-4-ol & $2438-10-0$ & $\mathrm{C}_{10} \mathrm{H}_{16} \mathrm{O}$ & 11.09 & Dai et al. [35] \\
\hline A52 & (5R)-5-Isopropenyl-2-methyl-2-cyclohexen-1-ol & $99-48-9$ & $\mathrm{C}_{10} \mathrm{H}_{16} \mathrm{O}$ & 0.12 & Dai et al. [35] \\
\hline A53 & Oct-1-en-3-ol & $3391-86-4$ & $\mathrm{C}_{8} \mathrm{H}_{16} \mathrm{O}$ & 2.57 & Dai et al. [35] \\
\hline A54 & $\alpha$-Phellandrene & $99-86-5$ & $\mathrm{C}_{10} \mathrm{H}_{16}$ & 1.66 & Dai et al. [35] \\
\hline
\end{tabular}

signaling pathway, demonstrated the multitarget and multipathway specialty of TCM in treating diseases.

Over 200 species of AAEO can be detected by gas chromatography-mass spectrometry (GC-MS) [34], mainly including terpenoids, ketones (aldehydes), alcohols (phenols), acids (esters), alkanes (alkenes), and other chemical constituents. In our study, $\beta$-caryophyllene, 1,8 cineole, terpinen-4-ol, neointermedeol, $\alpha$-thujone, borneol, and camphor had a relative content of the first 7 [14, 34-36]. 1,8-Cineole, camphor, and borneol accounted for the largest 


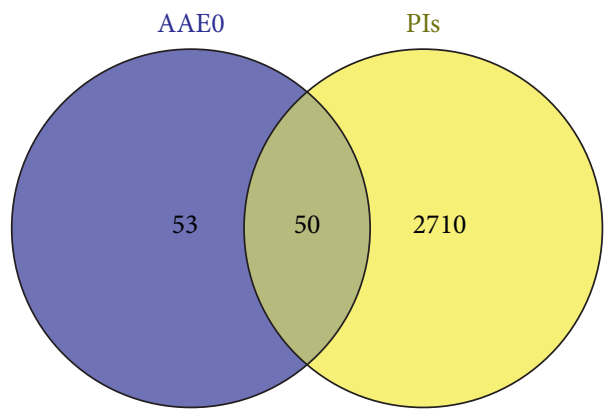

FIgURe 1: Venn diagram of targets' intersection of AAEO and PIs.

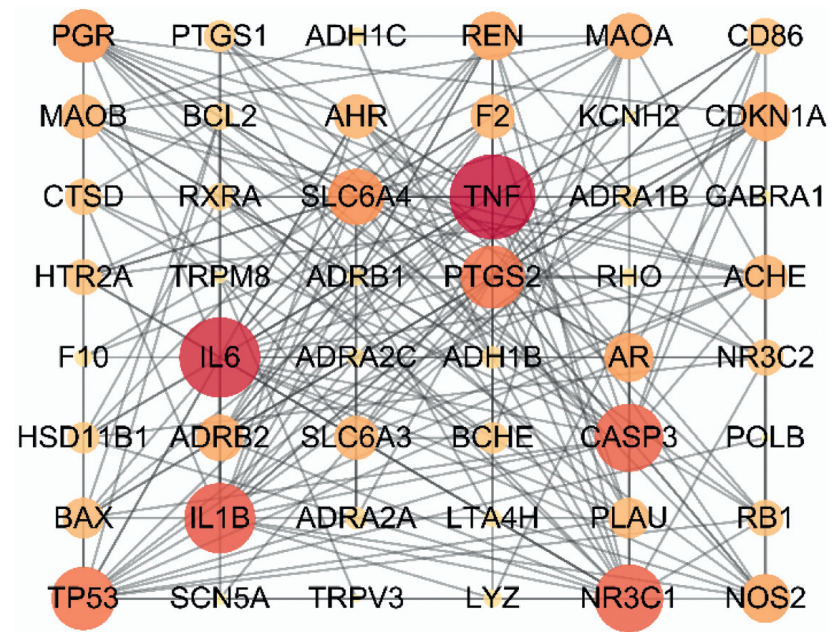

Figure 2: PPI network diagram. Protein-protein interactions $(P>0.7)$ of 50 overlapping targets.

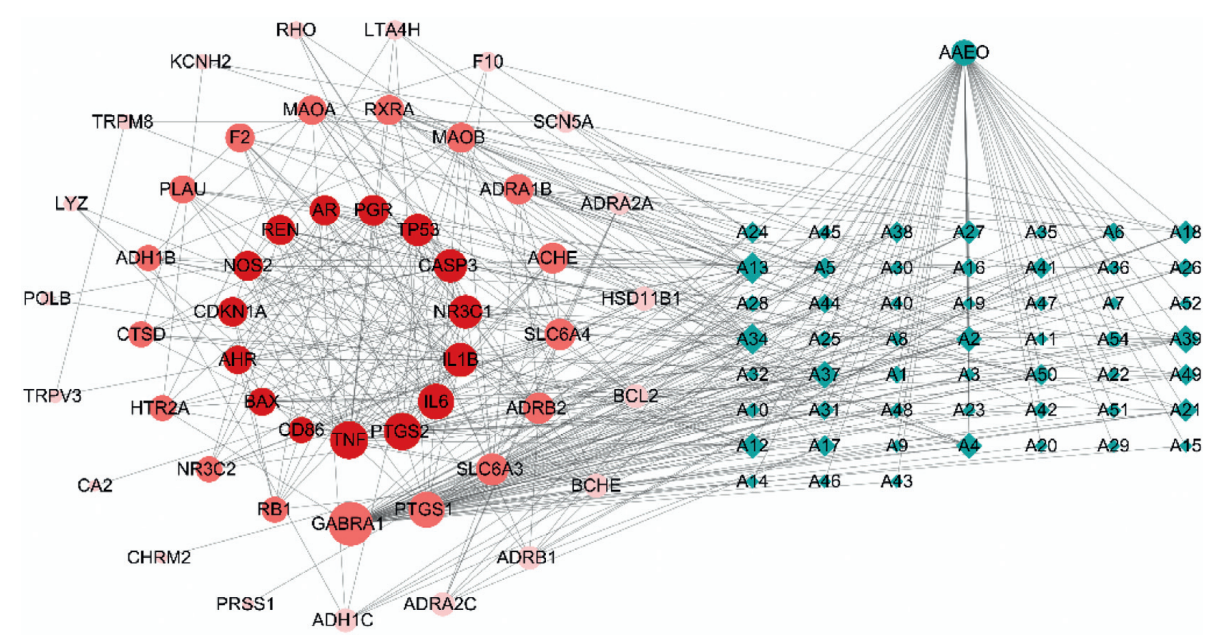

Figure 3: Compounds-overlapping targets network: the right square matrix green circle nodes represent 52 potential compounds $(2$ compounds (A33 and A53) have no associated targets) and the left circular nodes with gradual color represent 50 overlapping targets of AAEO and PIs. Larger size and deeper color of a node mean a greater degree.

proportion of AAEO [53]. In lipopolysaccharide0 (LPS-) induced cell and mouse inflammation experiments, 1,8 cineole alleviates LPS-induced vascular endothelial cell injury, obviously inhibits the production of the inflammatory mediator, increases the release of anti-inflammatory factor
IL10, and improves inflammatory symptoms [54]. Borneol significantly decreased the auricular swelling rate and pain threshold of rats by activating the p38-COX-2-PGE2 signaling pathway, which has significant analgesic and antiinflammatory effects on PDT of acne [55]. Numerous 


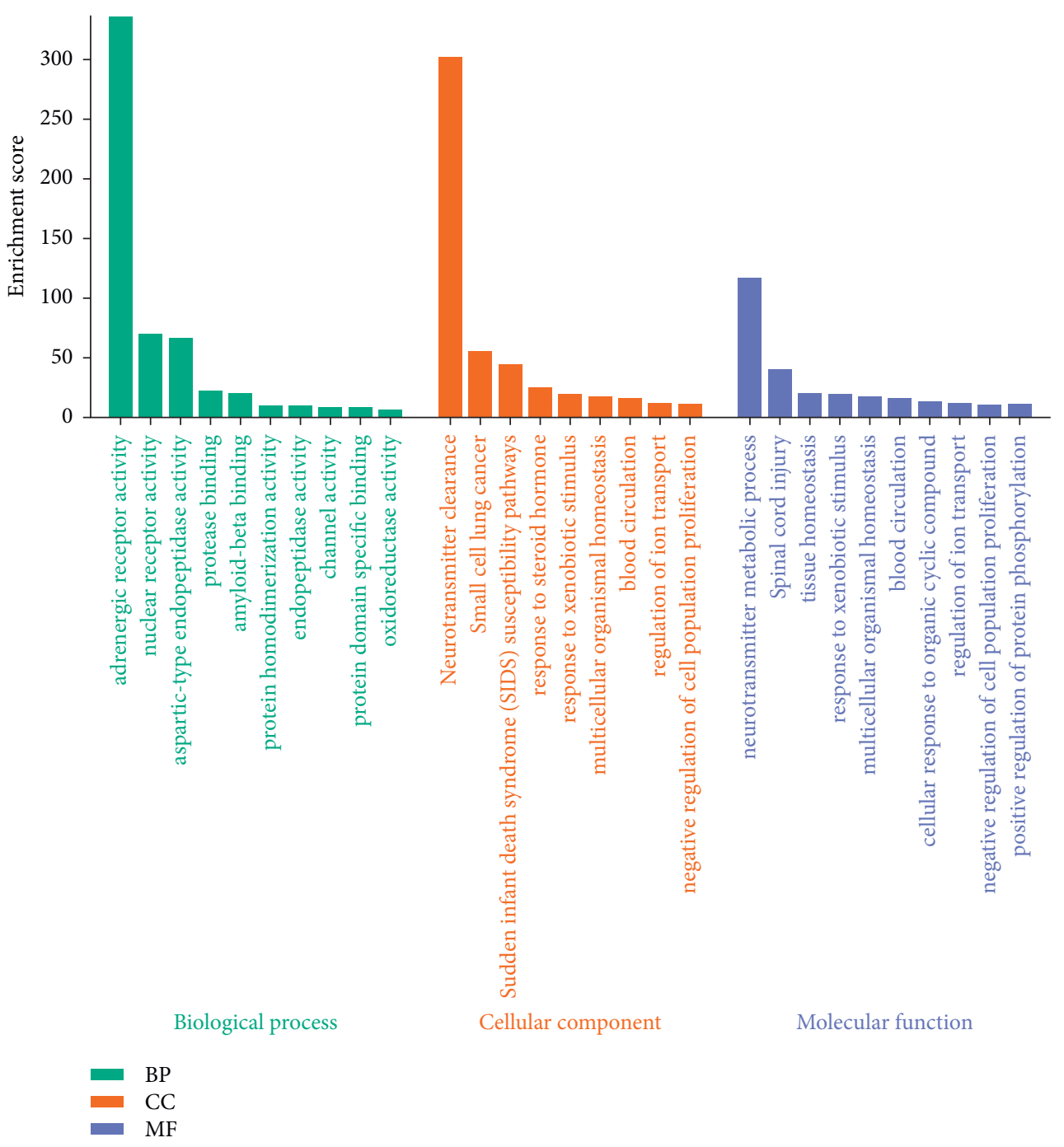

FIgURE 4: GO analysis.

investigations have shown various essential oils of several species containing camphor as the major component, exhibiting antimicrobial activity [56-59]. Also, the application of camphor to the skin was proved to increase local blood flow in the skin and muscle, induce both cold and warm sensations, and improve blood circulation [60]. More noteworthy is that the top three compounds of molecular docking score were neointermedeol, $\beta$-caryophyllene, and camphor. Neointermedeol has been shown to have antioxidant, antibacterial, and other biological activities [61, 62]. Recent studies have shown that caryophyllene can provide protection for animal cells and reduce proinflammatory mediators such as TNF- $\alpha$, IL- $1 \beta$, IL- 6 , and NF- $\kappa$ B, thereby improving the symptoms of inflammation and oxidative stress $[63,64]$.

The core network calculated by MCODE had 15 targets, mostly related to inflammation, oxidative stress, and apoptosis. TNF, IL6, IL-1 $\beta$, and PTGS2 participate in regulating inflammatory cascade reaction [65-68] and can be inhibited by inflammation in different levels by AAEO. TP53, BAX, and CASP3 regulate the apoptotic process and cell protection negatively $[69,70]$. KEGG Pathways enrichment analysis is mainly involved in PI3K-Akt signaling pathway, human immunodeficiency virus 1 infection, and human T-cell leukemia virus 1 infection, MAPK signaling pathway, and Wnt signaling pathway. The study found that the PI3KAkt pathway plays a great role in antiapoptosis and angiogenesis. The PI3K-Akt pathway phosphorylated Akt, and phosphorylated Akt first activated downstream factors Bad and Caspase-9 to play an antiapoptotic role and promote angiogenesis [71], and then phosphorylated Akt further regulated eNOS, which could promote the generation of $\mathrm{NO}$ [72], provide oxygen and nutrients for tissue recovery and mediate skin injury repair. Ischemic-reperfusion is recognized as the mechanism of PIs; the process includes oxidative stress, excessive release of oxygen free radicals, apoptosis, and activation of inflammatory cytokines [73]. The prediction results of our network pharmacology are mostly consistent with the progress of ischemic-reperfusion. MAPK signaling pathway is involved in the repair of PIs, increases the expression of Ras, c-Raf, MEK1, p-MEK1 protein, p-ERK1 protein, and MEK1 mRNA, promotes the 


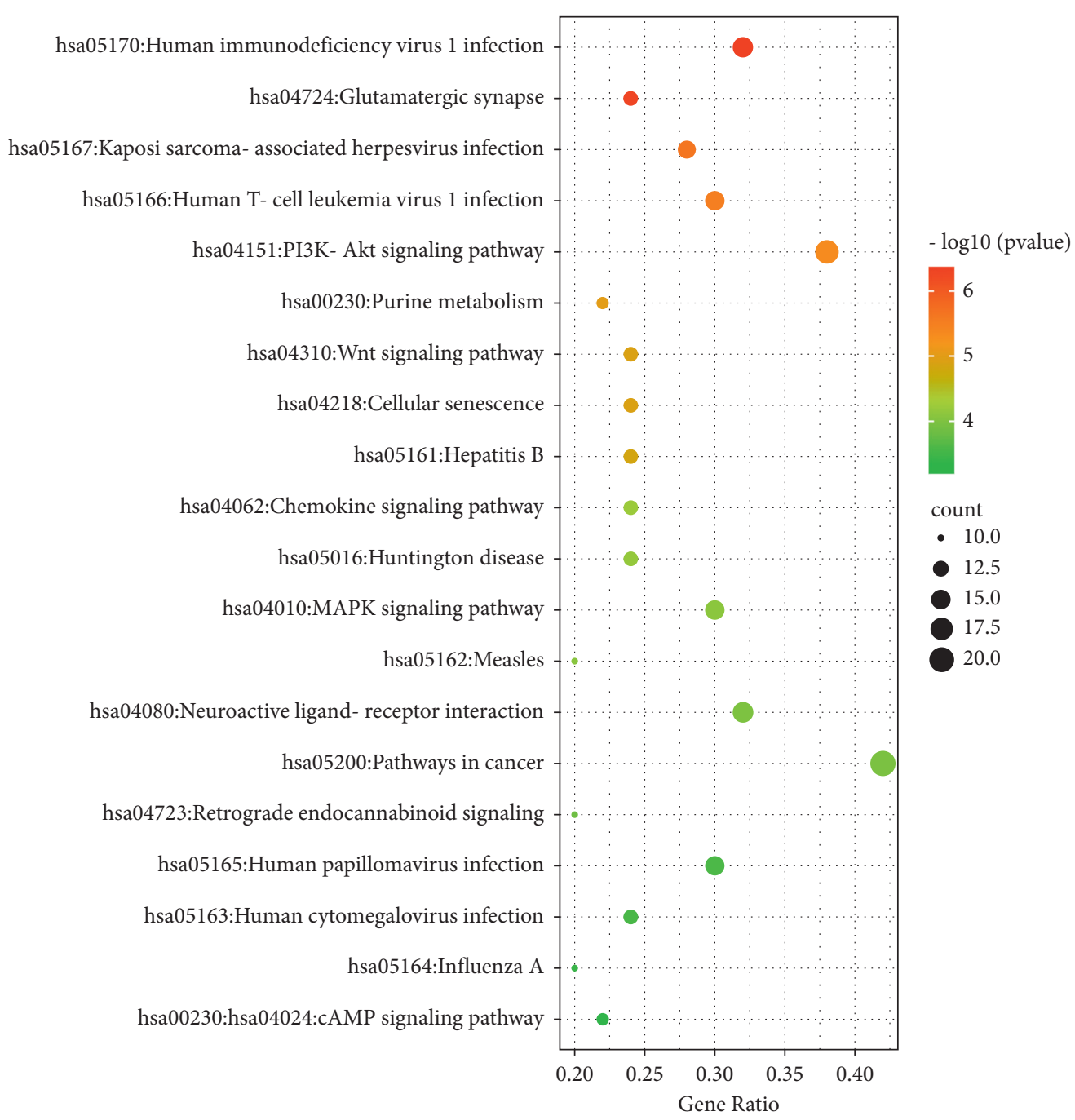

Figure 5: Top 20 enriched KEGG pathways.

TABLe 2: Vina score of compound-target docking (unit: kcal/mol).

\begin{tabular}{lcccccrc}
\hline ID & A27 & A1 & A51 & A4 & A11 & A6 \\
\hline TNF & -6 & -5.3 & -6.6 & -7.8 & -5.4 & -5.6 & -6.8 \\
PTGS2 & -6.6 & -7.1 & -6.8 & -7.2 & -6.4 & -6.2 & -5.3 \\
IL6 & -6.3 & -5.5 & -6.7 & -6.3 & -5.5 & -6.9 \\
IL1B & -6.8 & -5.6 & -5.5 & -7 & -5.3 & -5.6 & -5.9 \\
NR3C1 & -5.5 & -5.7 & -6.3 & -6.1 & -6.2 & -5.1 \\
CASP3 & -7 & -5.9 & -5.9 & -6.7 & -5.9 & -5.4 & -5.1 \\
TP53 & -7.4 & -5.8 & -6 & -7.1 & -6.1 & -5.8 \\
PGR & -7.8 & -6.7 & -6.4 & -7.6 & -6.6 & -6.2 & -6.5 \\
REN & -7.9 & -6.2 & -5.9 & -7.8 & -6.2 & -6.2 & -6.4 \\
NOS2 & -6.9 & -5.4 & -7 & -7.5 & -6.4 & -5.3 \\
\hline
\end{tabular}

proliferation of vascular endothelial cells, and accelerates microvascular regeneration and remodeling [74]. More studies have shown that the repair of pressure ulcers is highly correlated with the $\mathrm{Wnt} / \beta$-catenin signaling pathway regulating the proliferation and differentiation of epithelial cells, hair follicles, and sebaceous glands $[75,76]$.
The above arguments verify the accuracy of this network pharmacology prediction. Besides, the docking result showed that all selected core protein and ligand have a better affinity ( $\leq 5 \mathrm{kcal} / \mathrm{mol}$ ), and there were 15 docking scores $\geq 7 \mathrm{kcal} / \mathrm{mol}$, indicating strong binding affinity of the compound to docking protein [77]. The RMSD of the target 
Table 3: Docking parameters.

\begin{tabular}{|c|c|c|c|c|c|c|c|c|c|c|}
\hline \multirow{2}{*}{ Target } & \multirow{2}{*}{ PDB ID } & \multirow{2}{*}{ Ligand } & \multirow{2}{*}{ Cavity size } & \multicolumn{3}{|c|}{ Center } & \multicolumn{3}{|c|}{ Size } & \multirow{2}{*}{ RMSD } \\
\hline & & & & $X$ & $y$ & $z$ & $x$ & $y$ & $z$ & \\
\hline \multirow{7}{*}{ TNF } & \multirow{7}{*}{$1 \mathrm{D} 0 \mathrm{G}$} & A27 & 330 & 29 & 20 & 8 & 18 & 18 & 18 & 0.000 \\
\hline & & A1 & 791 & 9 & 38 & 47 & 16 & 16 & 16 & 0.098 \\
\hline & & A51 & 330 & 29 & 20 & 8 & 17 & 17 & 17 & 0.096 \\
\hline & & A4 & 330 & 29 & 20 & 8 & 18 & 18 & 18 & 0.000 \\
\hline & & A11 & 4587 & 23 & 55 & 16 & 35 & 16 & 28 & 0.585 \\
\hline & & A6 & 791 & 9 & 38 & 47 & 16 & 16 & 16 & 0.640 \\
\hline & & A3 & 1620 & 45 & 34 & 15 & 18 & 28 & 18 & 0.000 \\
\hline \multirow{7}{*}{ PTGS2 } & \multirow{7}{*}{ 1EQG } & A27 & 37209 & 48 & 34 & 189 & 35 & 35 & 35 & 0.548 \\
\hline & & A1 & 1809 & 73 & 23 & 195 & 24 & 26 & 28 & 0,104 \\
\hline & & A51 & 3589 & 22 & 28 & 203 & 26 & 29 & 24 & 0,103 \\
\hline & & $\mathrm{A} 4$ & 3589 & 22 & 28 & 203 & 26 & 29 & 24 & 0.000 \\
\hline & & A11 & 1809 & 73 & 23 & 195 & 24 & 26 & 28 & 0.591 \\
\hline & & A6 & 1809 & 73 & 23 & 195 & 24 & 26 & 28 & 0.645 \\
\hline & & A3 & 3589 & 22 & 28 & 203 & 26 & 29 & 24 & 0.000 \\
\hline \multirow{7}{*}{ IL6 } & \multirow{7}{*}{$4 \mathrm{O} 9 \mathrm{H}$} & A27 & 533 & -20 & 17 & 27 & 18 & 18 & 18 & 0.000 \\
\hline & & $\mathrm{A} 1$ & 533 & -20 & 17 & 27 & 16 & 16 & 16 & 0.103 \\
\hline & & A51 & 533 & -20 & 17 & 27 & 17 & 17 & 1 & 0.103 \\
\hline & & $\mathrm{A} 4$ & 533 & -20 & 17 & 27 & 18 & 18 & 18 & 0.000 \\
\hline & & A11 & 533 & -20 & 17 & 27 & 16 & 16 & 16 & 0.590 \\
\hline & & A6 & 533 & -20 & 17 & 27 & 16 & 16 & 16 & 0.664 \\
\hline & & A3 & 533 & -20 & 17 & 27 & 18 & 18 & 18 & 0.000 \\
\hline \multirow{7}{*}{$\operatorname{IL} 1 \beta$} & \multirow{7}{*}{$3 \mathrm{POK}$} & A27 & 987 & -15 & -17 & -8 & 18 & 18 & 18 & 0.000 \\
\hline & & A1 & 254 & 21 & 45 & 26 & 18 & 18 & 18 & 0.105 \\
\hline & & A51 & 987 & -15 & -17 & -8 & 23 & 17 & 17 & 0.103 \\
\hline & & $\mathrm{A} 4$ & 987 & -15 & -17 & -8 & 18 & 18 & 18 & 0.000 \\
\hline & & A11 & 199 & -25 & 5 & -7 & 16 & 16 & 16 & 0.592 \\
\hline & & A6 & 199 & -25 & 5 & -7 & 16 & 16 & 16 & 0.646 \\
\hline & & A3 & 987 & -15 & -17 & -8 & 18 & 18 & 18 & 0.000 \\
\hline & & A27 & 2172 & 31 & 38 & 81 & 28 & 32 & 35 & 0.000 \\
\hline & & A1 & 2172 & 31 & 38 & 81 & 28 & 32 & 35 & 0.000 \\
\hline & & A51 & 153 & 27 & 38 & 92 & 17 & 17 & 17 & 0.000 \\
\hline NR3C1 & $1 \mathrm{LAT}$ & $\mathrm{A} 4$ & 2172 & 31 & 38 & 81 & 28 & 32 & 35 & 0.000 \\
\hline & & A11 & 2172 & 31 & 38 & 81 & 28 & 32 & 35 & 0.000 \\
\hline & & A6 & 2172 & 31 & 38 & 81 & 28 & 32 & 35 & 0.000 \\
\hline & & $\mathrm{A} 3$ & 2172 & 31 & 38 & 81 & 28 & 32 & 35 & 0.000 \\
\hline & & A27 & 1834 & 3 & 4 & -25 & 18 & 31 & 18 & 0.000 \\
\hline & & $\mathrm{A} 1$ & 1834 & 3 & 4 & -25 & 17 & 31 & 17 & 0.104 \\
\hline & & A51 & 1834 & 3 & 4 & -25 & 17 & 31 & 17 & 0.103 \\
\hline CASP3 & 5JFT & $\mathrm{A} 4$ & 1834 & 3 & 4 & -25 & 18 & 31 & 18 & 0.000 \\
\hline & & A11 & 1834 & 3 & 4 & -25 & 17 & 31 & 17 & 1.153 \\
\hline & & A6 & 1834 & 3 & 4 & -25 & 16 & 31 & 16 & 0.646 \\
\hline & & A3 & 1834 & 3 & 4 & -25 & 16 & 31 & 16 & 0.592 \\
\hline & & A27 & 5969 & 36 & 3 & -12 & 26 & 35 & 31 & 0.000 \\
\hline & & $\mathrm{A} 1$ & 1644 & 23 & 18 & 12 & 30 & 16 & 16 & 0.272 \\
\hline & & A51 & 874 & 0 & -10 & 49 & 17 & 17 & 17 & 0.427 \\
\hline TP53 & 6WQX & $\mathrm{A} 4$ & 5969 & 36 & 3 & -12 & 26 & 35 & 31 & 0.000 \\
\hline & & A11 & 1644 & 23 & 18 & 12 & 30 & 16 & 16 & 1.153 \\
\hline & & A6 & 1644 & 23 & 18 & 12 & 30 & 16 & 16 & 0.586 \\
\hline & & A3 & 874 & 0 & -10 & 49 & 18 & 18 & 18 & 1.127 \\
\hline & & A27 & 617 & 43 & 34 & 30 & 18 & 18 & 18 & 0.000 \\
\hline & & $\mathrm{A} 1$ & 617 & 43 & 34 & 30 & 16 & 16 & 16 & 0.000 \\
\hline & & A51 & 631 & 23 & 5 & 73 & 17 & 17 & 17 & 0.000 \\
\hline PGR & $1 \mathrm{~A} 28$ & $\mathrm{~A} 4$ & 421 & 23 & 10 & 60 & 18 & 18 & 18 & 0.000 \\
\hline & & A11 & 617 & 43 & 34 & 30 & 17 & 17 & 17 & 0.000 \\
\hline & & A6 & 617 & 43 & 34 & 30 & 16 & 16 & 16 & 0.000 \\
\hline & & A3 & 617 & 43 & 34 & 30 & 16 & 16 & 16 & 0.000 \\
\hline
\end{tabular}


TABle 3: Continued.

\begin{tabular}{|c|c|c|c|c|c|c|c|c|c|c|}
\hline \multirow{2}{*}{ Target } & \multirow{2}{*}{ PDB ID } & \multirow{2}{*}{ Ligand } & \multirow{2}{*}{ Cavity size } & \multicolumn{3}{|c|}{ Center } & \multicolumn{3}{|c|}{ Size } & \multirow{2}{*}{ RMSD } \\
\hline & & & & $X$ & $y$ & $z$ & $x$ & $y$ & $z$ & \\
\hline \multirow{7}{*}{ REN } & \multirow{7}{*}{$3 \mathrm{OWN}$} & A27 & 11842 & 9 & -14 & -30 & 35 & 35 & 33 & 0.000 \\
\hline & & $\mathrm{A} 1$ & 1910 & 20 & -1 & -18 & 26 & 16 & 16 & 0.107 \\
\hline & & A51 & 1834 & 3 & 4 & -25 & 17 & 31 & 17 & 0.106 \\
\hline & & $\mathrm{A} 4$ & 1910 & 20 & -1 & -18 & 26 & 18 & 18 & 0.000 \\
\hline & & A11 & 1682 & -10 & -28 & -37 & 17 & 23 & 17 & 1.156 \\
\hline & & A6 & 1682 & -10 & -28 & -37 & 16 & 23 & 22 & 0.648 \\
\hline & & A3 & 11842 & 9 & -14 & -30 & 35 & 35 & 33 & 0.594 \\
\hline \multirow{7}{*}{ NOS2 } & \multirow{7}{*}{$1 \mathrm{M} 7 \mathrm{Z}$} & A27 & 3650 & 5 & 33 & 11 & 30 & 27 & 30 & 0.000 \\
\hline & & $\mathrm{A} 1$ & 3650 & 5 & 33 & 11 & 30 & 27 & 30 & 0.103 \\
\hline & & A51 & 3650 & 5 & 33 & 11 & 30 & 27 & 30 & 0.102 \\
\hline & & $\mathrm{A} 4$ & 3650 & 5 & 33 & 11 & 30 & 27 & 30 & 0.000 \\
\hline & & A11 & 3650 & 5 & 33 & 11 & 30 & 27 & 30 & 1.152 \\
\hline & & A6 & 3650 & 5 & 33 & 11 & 30 & 27 & 30 & 0.645 \\
\hline & & A3 & 3650 & 5 & 33 & 11 & 30 & 27 & 30 & 0.590 \\
\hline
\end{tabular}

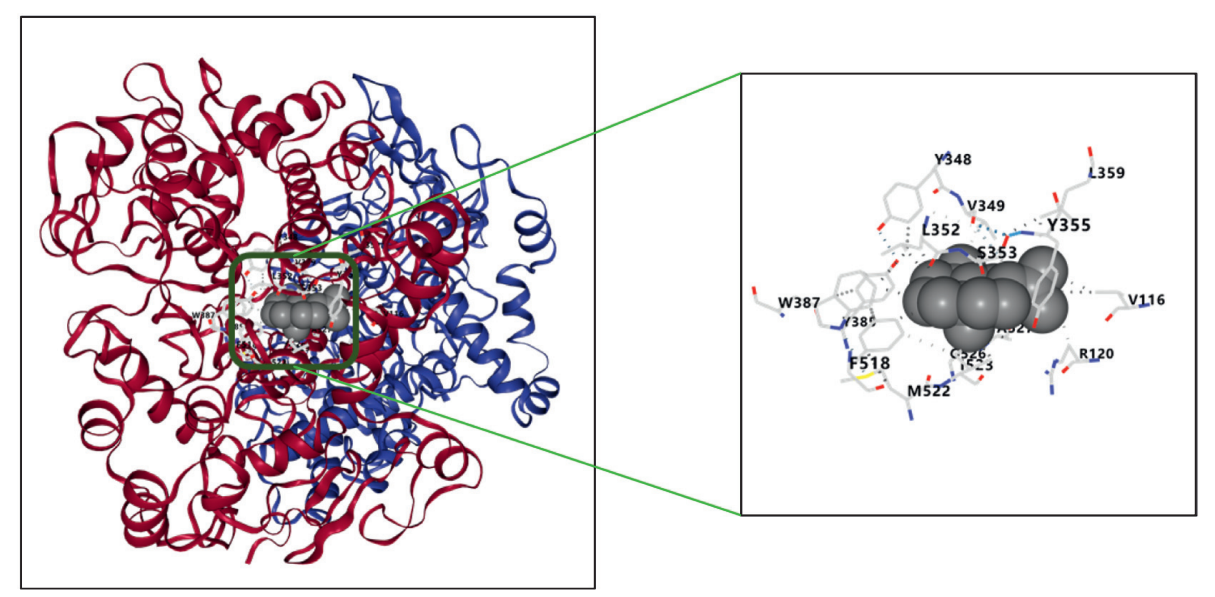

A4 \& 1EQG

(a)

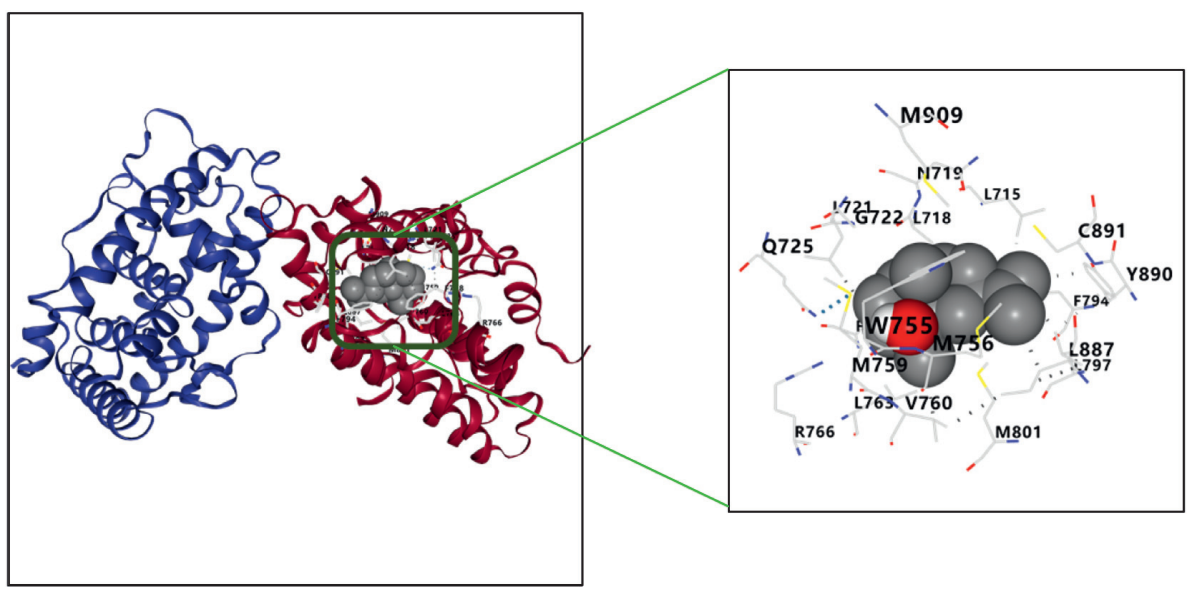

A4 \& $1 \mathrm{~A} 28$

(b)

Figure 6: Continued. 


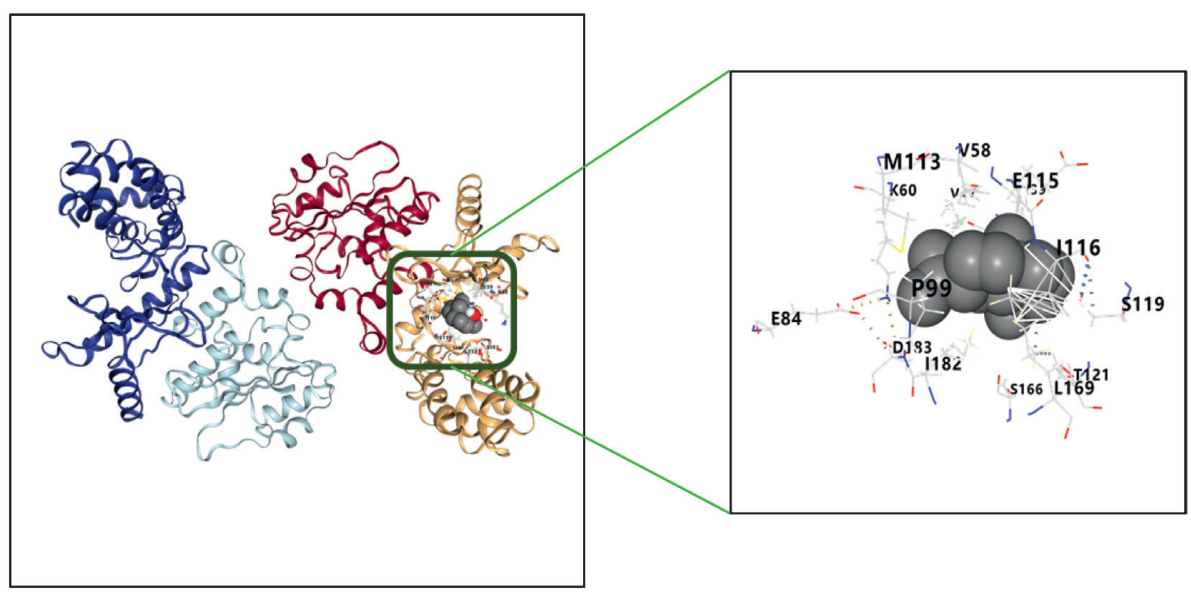

A4 \& 6WQX

(c)

FIGURE 6: $(\mathrm{a}-\mathrm{c})$ Docking results of compound A4-neointermedeol and PTGS2 (1EQG), PGR (1A28), and TP53 (6WQX), respectively.

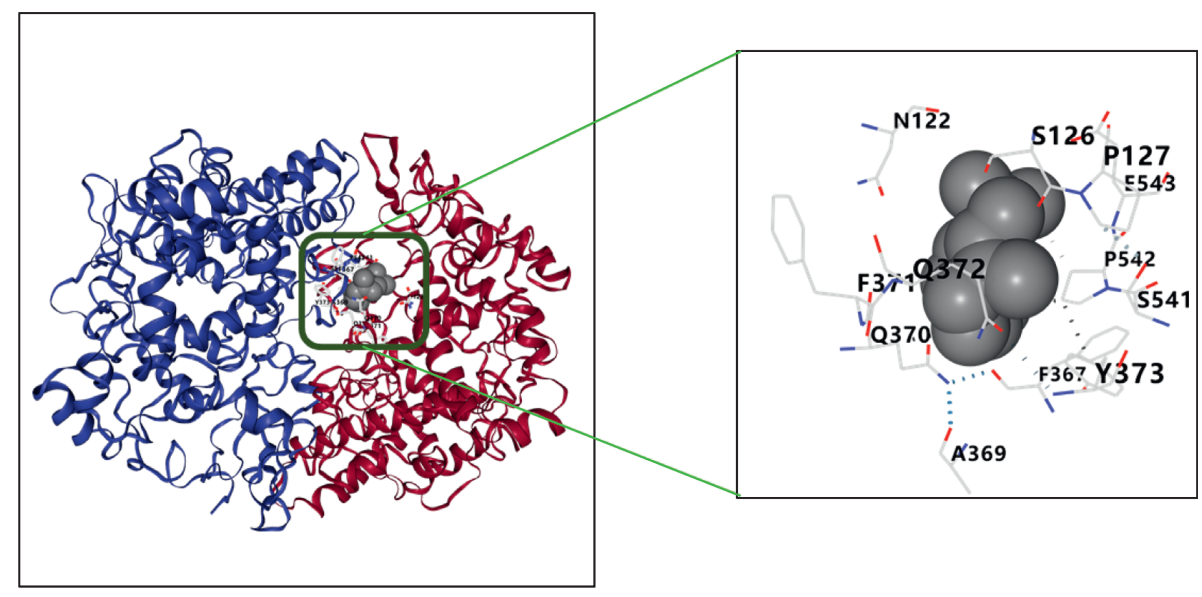

A27 \& 1EQG

(a)

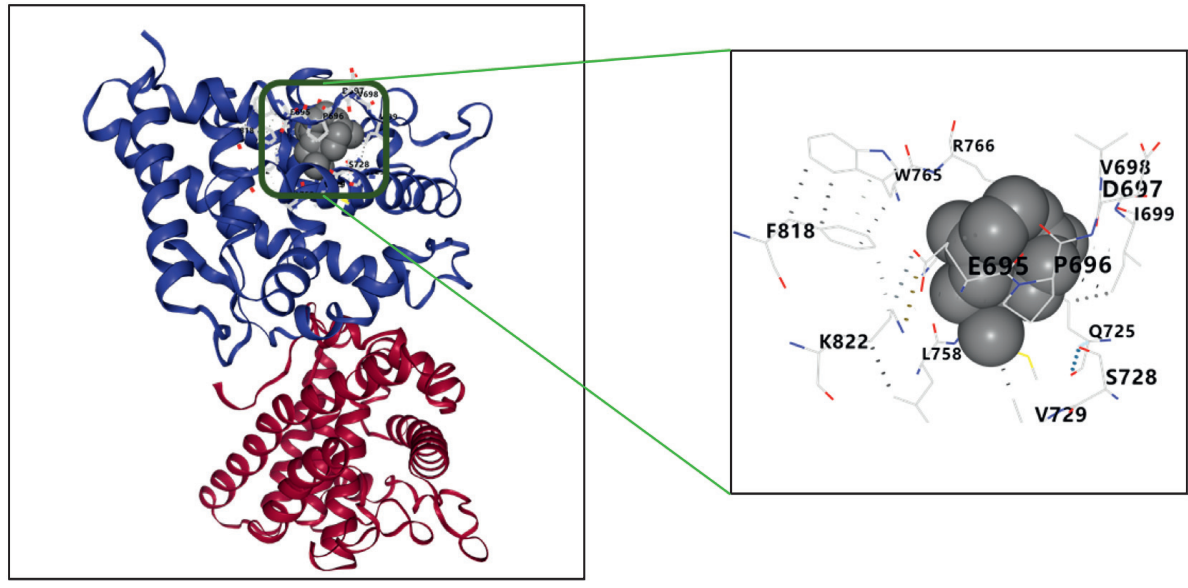

A27 \& 1 A28

(b)

Figure 7: Continued. 


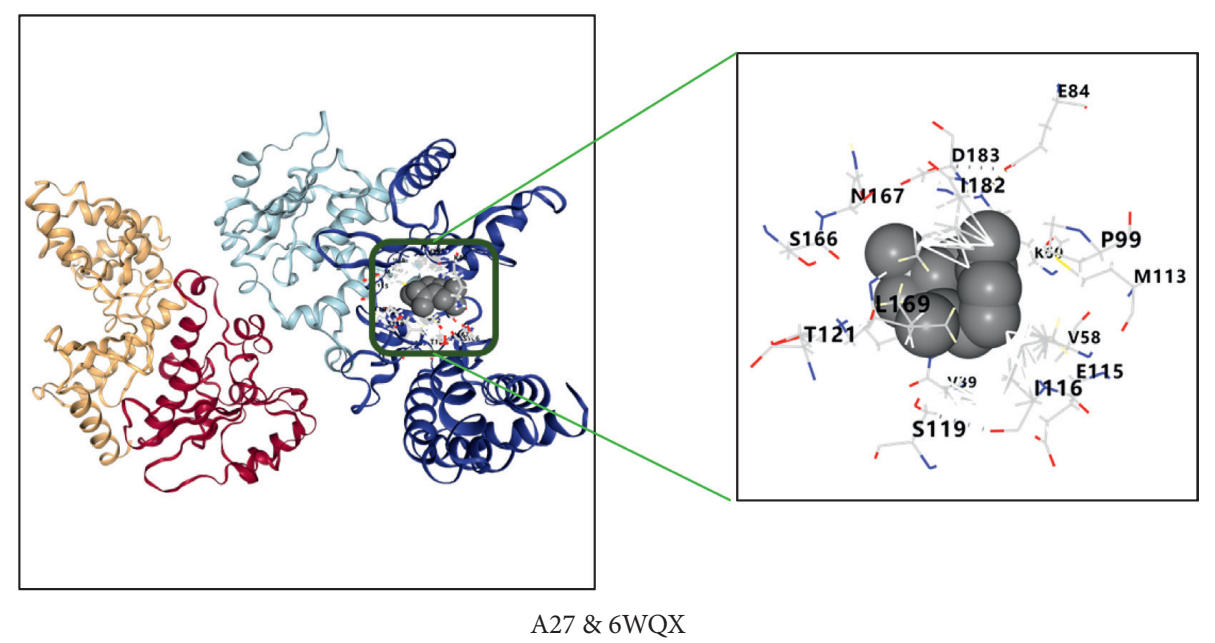

(c)

FIGURe 7: (a-c) Docking results of compound A27 $\beta$-caryophyllene and PTGS2 (1EQG), PGR (1A28), and TP53 (6WQX), respectively.

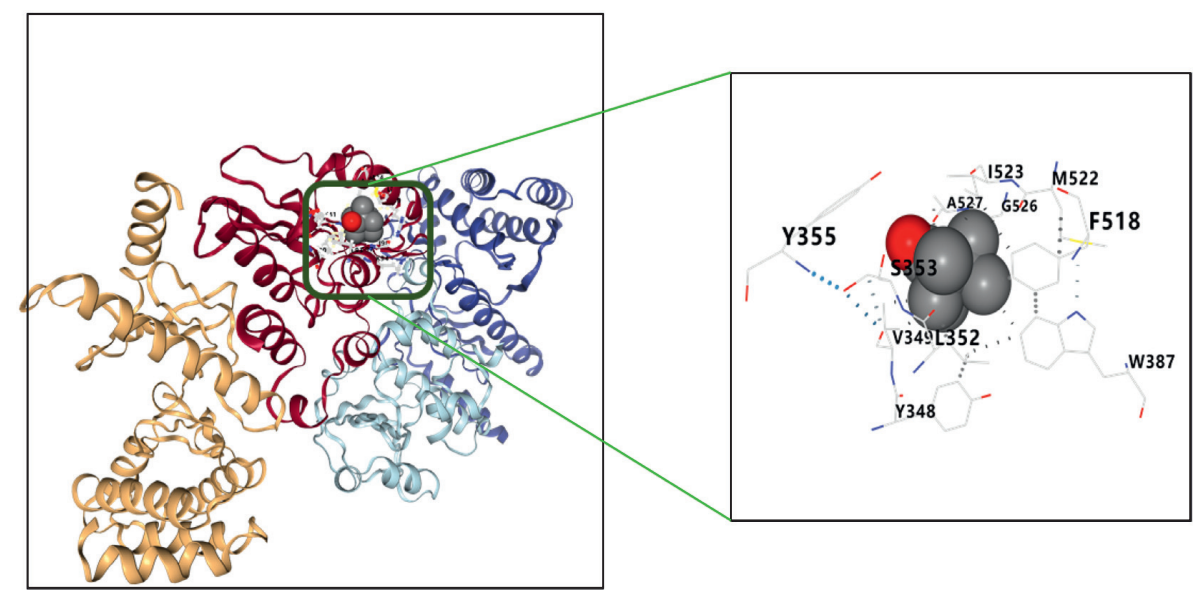

A3 \& $1 \mathrm{EQG}$

(a)

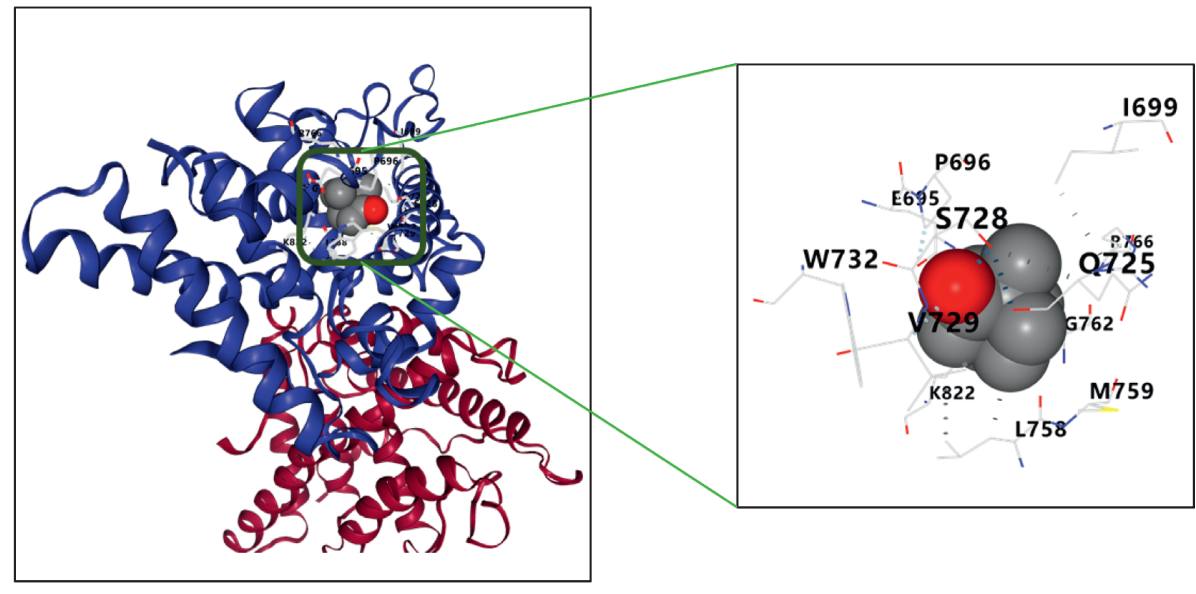

A3 \& 1 A28

(b)

Figure 8: Continued. 


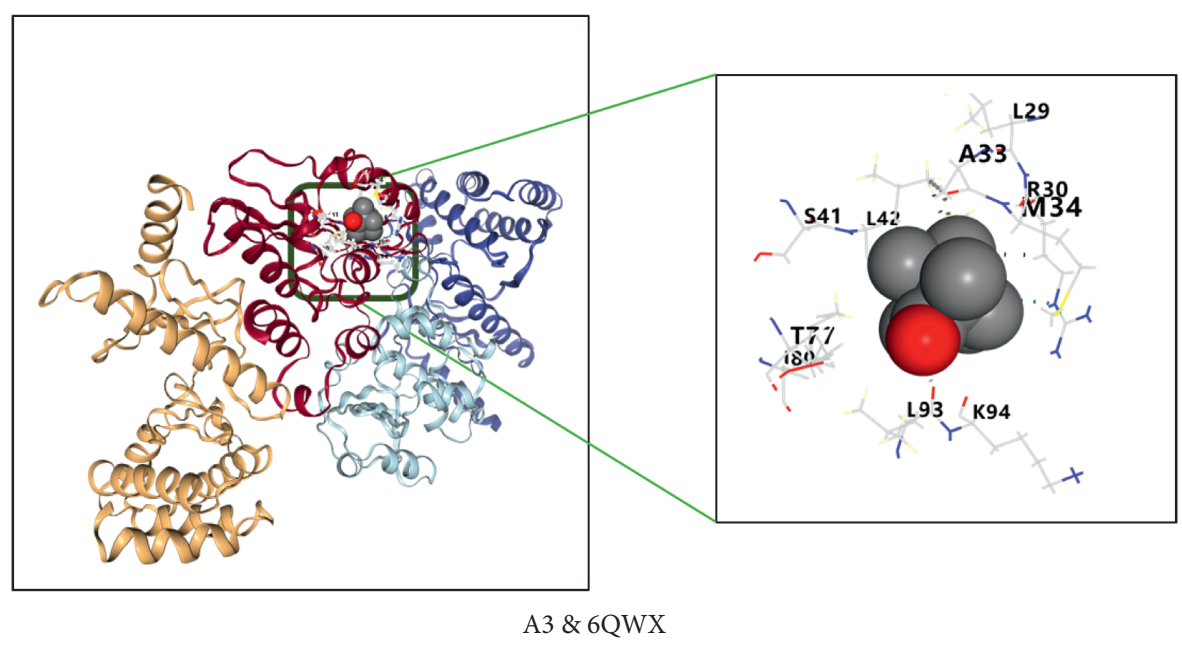

(c)

FIGURE 8: (a-c) Docking results of compound A3 terpinen-4-ol and PTGS2 (1EQG), PGR (1A28), and TP53 (6WQX), respectively.

protein is less than $2 \AA$, which indicates that the docking method and parameter setting are reasonable and can be used for the next docking with components [78].

In addition, study showed that AAEO dose-dependently inhibits inflammatory mediators, such as NO, PGE2, TNF- $\alpha$, IL-6, IL-10, IFN- $\beta$, and MCP-1 [79]. In the experiment of AAEO in anti-inflammatory and blood stasis animals, the effect of the lowest dose of skin administration $(0.25 \mathrm{~mL} / \mathrm{kg})$ was equivalent to that oral administration of the middle dose $(0.50 \mathrm{~mL} / \mathrm{kg})$ [80]. PTGS2 with the highest docking scores is a biomarker of iron death; it can inhibit the expression of inflammatory factors and apoptosis [81-83]. The future research direction can explore the way of administration, dosage, and iron death mechanism pathway.

The limitation of this study is that we have not conducted clinical or animal experiments as certification; further studies will validate the potential key targets and pathways predicted and explore the mechanism of effective components of the essential oil from Artemisia argyi in preventing and treating PIs by combining molecular biology and pathophysiology.

\section{Conclusion}

In conclusion, in this study, the potential targets and regulatory molecular mechanisms of AAEO in the treatment of PIs were analyzed by network pharmacology and molecular docking. In total, 54 active components and 50 potential targets were screened, mainly involving PI3K-Akt signaling pathway, pathway in cancer, PI3K-Akt signaling pathway, human immunodeficiency virus 1 infection, MAPK signaling pathway, and $\mathrm{Wnt}$ signaling pathway, revealing that AAEO may play a role in the treatment of PIs by reducing inflammation, inhibiting apoptosis and oxidative stress, and showing the characteristics of multitarget and multipathway. Our study provides a basis for the mechanism and further research direction of AAEO in treating PIs by combining literature research, network analysis, and molecular docking.

\section{Data Availability}

All data generated or analyzed during this study are included in this article.

\section{Disclosure}

Shu-ting $\mathrm{Lu}$ is the first author.

\section{Conflicts of Interest}

All authors declare that they have no conflicts of interest regarding the publication of this paper.

\section{Authors' Contributions}

Ying-tao Lai and Ling-han Zhou designed the study. Shuting $\mathrm{Lu}$ wrote the manuscript and made the tables and figures. Lu-lu Tang was responsible for the translation and correction of the article. Yi-fan Duan and Lan-xing Liu performed network analysis.

\section{Acknowledgments}

This work was supported by Guangzhou University of Traditional Chinese Medicine First Affiliated Hospital Innovation Hospital Project (No. 211010010203).

\section{References}

[1] L. E. Edsberg, J. M. Black, M. Goldberg, L. McNichol, L. Moore, and M. Sieggreen, "Revised national pressure ulcer advisory panel pressure injury staging system," The Journal of Wound, Ostomy and Continence Nursing, vol. 43, no. 6, pp. 585-597, 2016.

[2] W. V. Padula and B. A. Delarmente, "The national cost of hospital-acquired pressure injuries in the United States," International Wound Journal, vol. 16, no. 3, pp. 634-640, 2019. 
[3] D. Anthony, D. Alosoumi, and R. Safari, "Prevalence of pressure ulcers in long-term care: a global review," Journal of Wound Care, vol. 28, no. 11, pp. 702-709, 2019.

[4] N. Kučišec-Tepeš, "Characteristic features of pressure ulcer infection," Acta Medica Croatica, vol. 70, no. 1, pp. 45-51, 2016.

[5] H. Zolfagharnezhad, H. Khalili, M. Mohammadi, S. Niknam, and A. Vatanara, "Topical nifedipine for the treatment of pressure ulcer: a randomized, placebo-controlled clinical trial," American Journal of Therapeutics, vol. 28, no. 1, pp. e41-e51, 2021.

[6] T. Y. Yang and S. H. Shin, "Effect of soft silicone foam dressings on intraoperatively acquired pressure injuries: a randomized study in patients undergoing spinal surgery," Wound Management and Prevention, vol. 66, no. 11, 2020.

[7] S. Perez-Lopez, M. Perez-Basterrechea, J. M. Garcia-Gala, E. Martinez-Revuelta, A. Fernandez-Rodriguez, and M. Alvarez-Viejo, "Stem cell and tissue engineering approaches in pressure ulcer treatment," The journal of spinal cord medicine, pp. 1-10, 2021.

[8] D. Seidel, M. Storck, H. Lawall et al., "Negative pressure wound therapy compared with standard moist wound care on diabetic," BMJ Open, vol. 10, Article ID e26345, 2020.

[9] J. S. Mervis and T. J. Phillips, "Pressure ulcers: prevention and management," Journal of the American Academy of Dermatology, vol. 81, no. 4, pp. 893-902, 2019.

[10] L. Duan, C. Zhang, C. Zhang, Z. Xue, Y. Zheng, and L. Guo, "Green extraction of phenolic acids from Artemisia argyi leaves by tailor-made ternary deep eutectic solvents," Molecules, vol. 24, no. 15, p. 2842, 2019.

[11] K. Wang, Z. Wan, and J. Zhou, "Network pharmacology analysis of volatile components from Artemisia argyi leaves on knee joint disease," World Traditional Chinese Medicine, pp. 1-13, 2021.

[12] Yu. Yuan, "Study on prescription rules and mechanism of 《gynecologic treasures》," in Treating Pregnancy Based on Data Mining TechnologyShanxi University of Traditional Chinese Medicine, Taiyuan, China, 2021.

[13] L.-B. Zhang, X.-N. Nie, J.-J. Chang, F.-L. Wang, and J.-L. Liu, "Nitric oxide production inhibitory eudesmane-type sesquiterpenoids from Artemisia," Chemistry and Biodiversity, vol. 17, no. 7, Article ID e2000238, 2020.

[14] X. Guan, D. Ge, S. Li, K. Huang, J. Liu, and F. Li, "Chemical composition and antimicrobial activities of artemisia argyi Lévl. et Vant essential oils extracted by simultaneous distillation-extraction, subcritical extraction and hydrodistillation," Molecules, vol. 24, no. 3, 2019.

[15] L.-L. Chen, H.-J. Zhang, J. Chao, and J.-F. Liu, "Essential oil of Artemisia argyi suppresses inflammatory responses by inhibiting JAK/STATs activation," Journal of Ethnopharmacology, vol. 204, pp. 107-117, 2017.

[16] X. Bao, H. Yuan, C. Wang, J. Liu, and M. Lan, "Antitumor and immunomodulatory activities of a polysaccharide from Artemisia argyi," Carbohydrate Polymers, vol. 98, no. 1, pp. 1236-1243, 2013.

[17] B. Ku, M. Jun, J. H. Lee et al., "Short-Term efficacy of pulsed radiofrequency thermal stimulation on acupoints for chronic low back pain: a preliminary study of a randomized, singleblinded, placebo-controlled trial," Evidence-Based Complementary and Alternative Medicine, vol. 2018, Article ID 4510909, 2018.

[18] Y. Li, C. Sun, J. Kuang, C Ji, and J Wu, "The effect of moxibustion stimulation on local and distal skin temperature in healthy subjects," Evidence-Based Complementary and Alternative Medicine, vol. 2019, Article ID 3185987, 2019.

[19] L. Ha, M. Yu, Z. Yan, Z. Rui, and B. Zhao, "Effects of moxibustion and moxa smoke on behavior changes and energy metabolism," Evidence-Based Complementary and Alternative Medicine, vol. 2019, Article ID 9419567, 2019.

[20] A. L. Hopkins, "Network pharmacology: the next paradigm in drug discovery," Nature Chemical Biology, vol. 4, no. 11, pp. 682-690, 2008.

[21] D. C. Hao and P. G. Xiao, "Network pharmacology: a Rosetta Stone for traditional Chinese medicine," Drug Development Research, vol. 75, no. 5, pp. 299-312, 2014.

[22] M. A. Yildirim, K. I. Goh, M. E. Cusick, A. L. Barabási, and M. Vidal, "Drug-target network," Nature Biotechnology, vol. 25, no. 10, pp. 1119-1126, 2007.

[23] L. Shao, "Exploring traditional Chinese medicine by a novel therapeutic concept of network target," Chinese Journal of Integrative Medicine, vol. 22, no. 9, 2016.

[24] S. Liu, Q. Li, F. Liu et al., "Uncovering the mechanism of curcuma in the treatment of ulcerative colitis based on network pharmacology, molecular docking technology, and experiment verification," Evidence-Based Complementary and Alternative Medicine, vol. 2021, pp. 1-14, 2021.

[25] Y. S. Lee, S. Y. Lee, S. Y. Park, S. W. Lee, K. W. Hong, and C. D. Kim, "Cilostazol add-on therapy for celecoxib synergistically inhibits proinflammatory cytokines by activating IL10 and SOCS3 in the synovial fibroblasts of patients with rheumatoid arthritis," Inflammopharmacology, vol. 27, no. 6, pp. 1205-1216, 2019.

[26] N. Yamada, T. Karasawa, T. Wakiya et al., "Iron overload as a risk factor for hepatic ischemia-reperfusion injury in liver transplantation: potential role of ferroptosis," American Journal of Transplantation, vol. 20, no. 6, pp. 1606-1618, 2020.

[27] X. Li, N. Ma, J. Xu et al., "Targeting ferroptosis: pathological mechanism and treatment of ischemia-reperfusion injury," Oxidative Medicine and Cellular Longevity, vol. 2021, Article ID 1587922, 2021.

[28] P.-Z. Han, D.-H. Cao, X.-L. Zhang, Z.-J. Ren, and Q. Wei, "Association between TP53 gene codon72 polymorphism and prostate cancer risk," Medicine, vol. 98, no. 25, Article ID e16135, 2019.

[29] R. Bologna-Molina, V. Pereira-Prado, C. Sánchez-Romero, R. González-González, and A. Mosqueda-Taylor, "Primordial odontogenic tumor: a systematic review," Medicina Oral, Patología Oral y Cirugía Bucal, vol. 25, no. 3, pp. e388-e394, 2020.

[30] R. M. Ghali, M. A. Al-Mutawa, B. H. Ebrahim et al., "Progesterone receptor (PGR) gene variants associated with breast cancer and associated features: a case-control study," $\mathrm{Pa}$ thology and Oncology Research, vol. 26, no. 1, pp. 141-147, 2020.

[31] S. Chiang, W. Samore, L. Zhang et al., "PGR gene fusions identify a molecular subset of uterine epithelioid leiomyosarcoma with rhabdoid features," The American Journal of Surgical Pathology, vol. 43, no. 6, pp. 810-818, 2019.

[32] K. Beyfuss and D. A. Hood, "A systematic review of p53 regulation of oxidative stress in skeletal muscle," Redox Report, vol. 23, no. 1, pp. 100-117, 2018.

[33] Y. Liu, Y. He, F. Wang et al., "From longevity grass to contemporary soft gold: explore the chemical constituents, pharmacology, and toxicology of Artemisia argyi H.Lév. \& vaniot essential oil," Journal of Ethnopharmacology, vol. 279, Article ID 114404, 2021. 
[34] Z. Xuelin, X. Chen, and Y. Wu, "Research progress on the chemical constituents and pharmacological activities of volatile oil of AiYe (Artemisia argyi)," Chinese Archives of Traditional Chinese Medicine, vol. 45, no. 10, pp. 2417-2424, 2021.

[35] W. Dai, Y. Li, Q. Mei, X. Wang, and P. Dong, "GC-MS analysis of volatile oil of mugwort from 12 different area," Journal of Chinese Medicinal Materials, vol. 38, no. 12, pp. 2502-2506, 2015.

[36] Y. Gu, Z. Liang, S. Chen, L. Zhou, L. Yang, and Y. Zeng, "Analysis of volatile oil from Artemisia argyi in Guangxi by GC-MS," South China Agriculture, vol. 14, no. 28, pp. 13-15, 2020.

[37] Y. Yang, Y. Li, J. Wang et al., "Systematic investigation of ginkgo biloba leaves for treating cardio-cerebrovascular diseases in an animal model," ACS Chemical Biology, vol. 12, no. 5, pp. 1363-1372, 2017.

[38] S. Kim, J. Chen, T. Cheng et al., "PubChem in 2021: new data content and improved web interfaces," Nucleic Acids Research, vol. 49, no. D1, pp. D1388-D1395, 2021.

[39] D. S. Wishart, C. Knox, A. C. Guo et al., "DrugBank: a comprehensive resource for in silico drug discovery and exploration," Nucleic acids research, vol. 34, pp. D668-D672, 2006.

[40] X. Wang, Y. Shen, S. Wang et al., "PharmMapper 2017 update: a web server for potential drug target identification with a comprehensive target pharmacophore database," Nucleic Acids Research, vol. 45, no. W1, pp. W356-W360, 2017.

[41] B. Liang, C. Li, and J. Zhao, "Identification of key pathways and genes in colorectal cancer using bioinformatics analysis," Medical Oncology, vol. 33, no. 10, p. 111, 2016.

[42] M. Franz, C. T. Lopes, G. Huck, Y. Dong, O. Sumer, and G. D. Bader, "Cytoscape.js: a graph theory library for visualisation and analysis," Bioinformatics, vol. 32, no. 2, pp. 309-311, 2016.

[43] W. Wang, Z. Wan, J. Zhou et al., "Network pharmacological of volatile components of Artemisia Argyi on knee osteoarthrosis," World Chinese Medicine, vol. 16, no. 20, pp. 2975-2979, 2021.

[44] F. Li, P. Guo, K. Dong, P. Guo, H. Wang, and X. Lv, "Identification of key biomarkers and potential molecular mechanisms in renal cell carcinoma by bioinformatics analysis," Journal of Computational Biology, vol. 26, no. 11, pp. 1278-1295, 2019.

[45] D. Bu, H. Luo, P. Huo et al., "KOBAS-i: intelligent prioritization and exploratory visualization of biological functions for gene enrichment analysis," Nucleic acids research, vol. 49, no. W1, pp. W317-W325, 2021.

[46] D. W. Huang, B. T. Sherman, and R. A. Lempicki, "Systematic and integrative analysis of large gene lists using DAVID bioinformatics resources," Nature Protocols, vol. 4, no. 1, pp. 44-57, 2009.

[47] Q. Huang, R. Liu, J. Liu, Q. Huang, S. Liu, and Y. Jiang, "Integrated network pharmacology analysis and experimental validation to reveal the mechanism of anti-insulin resistance effects of moringa oleifera seeds," Drug Design, Development and Therapy, vol. 14, pp. 4069-4084, 2020.

[48] X. Ma, Y. Du, X. Zhu, Z. Feng, C. Chen, and J. Yang, "Evaluation of an ionic liquid chiral selector based on clindamycin phosphate in capillary electrophoresis," Analytical and Bioanalytical Chemistry, vol. 411, no. 22, pp. 5855-5866, 2019.

[49] Y. Liu, M. Grimm, W.-t. Dai, M.-c. Hou, Z.-X. Xiao, and Y. Cao, "CB-Dock: a web server for cavity detection-guided protein-ligand blind docking," Acta Pharmacologica Sinica, vol. 41, no. 1, pp. 138-144, 2020.

[50] Y. G. Tao, X. F. Huang, J. Y. Wang, M. R. Kang, L. J. Wang, and S. X. Xian, "Exploring molecular mechanism of huangqi in treating heart failure using network pharmacology," Evidence-based Complementary and Alternative Medicine, vol. 2020, Article ID 6473745, 2020.

[51] J. J. Sutherland, R. K. Nandigam, J. A. Erickson, and M. Vieth, "Lessons in molecular recognition. 2. Assessing and improving cross-docking accuracy," Journal of Chemical Information and Modeling, vol. 47, no. 6, pp. 2293-2302, 2007.

[52] X. Zhang, X. Chen, and Y. Wu, "Research progress on chemical constituents and pharmacologiacal activities of volatile oil of AiYe (artemisia argyi )," Chinese Archives of Traditional Chinese Medicine, vol. 39, no. 5, pp. 111-118, 2021.

[53] X. Song, X. Wen, and J. He, "Phytochemical components and biological activities of Artemisia argyi," Journal of Functional Foods, vol. 52, 2019.

[54] K.-G. Linghu, G.-P. Wu, L.-Y. Fu et al., "1,8-Cineole ameliorates LPS-induced vascular endothelium dysfunction in mice via PPAR- $\gamma$ dependent regulation of NF- $\kappa$ B, " Frontiers in Pharmacology, vol. 10, p. 178, 2019.

[55] J. Ji, R. Zhang, H. Li, J. Zhu, Y. Pan, and Q. Guo, “Analgesic and anti-inflammatory effects and mechanism of action of borneol on photodynamic therapy of acne," Environmental Toxicology and Pharmacology, vol. 75, Article ID 103329, 2020.

[56] F. J. Hammerschmidt, A. M. Clark, F. M. Soliman, E. S elKashoury, M. M Abd el-Kawy, and A. M el-Fishawy, "Chemical composition and antimicrobial activity of essential oils of Jasonia candicans and J. Montana," Planta Medica, vol. 59, no. 1, pp. 68-70, 1993.

[57] P. Magiatis, A. L. Skaltsounis, I. Chinou, and S. A Haroutounian, "Chemical composition and in-vitro antimicrobial activity of the essential oils of three Greek Achillea species." Zeitschrift fur Naturforschung. C, Journal of Biosciences, vol. 57, no. 3-4, pp. 287-290, 2002.

[58] Z. Shunying, Y. Yang, Y. Huaidong, Y Yue, and Z Guolin, "Chemical composition and antimicrobial activity of the essential oils of Chrysanthemum indicum," Journal of Ethnopharmacology, vol. 96, no. 1-2, pp. 151-158, 2005.

[59] F. Juteau, V. Masotti, J. M. Bessière, M. Dherbomez, and J. Viano, "Antibacterial and antioxidant activities of Artemisia annua essential oil," Fitoterapia, vol. 73, no. 6, pp. 532-535, 2002.

[60] T. Kotaka, S. Kimura, M. Kashiwayanagi, and J. Iwamoto, "Camphor induces cold and warm sensations with increases in skin and muscle blood flow in human," Biological and Pharmaceutical Bulletin, vol. 37, no. 12, pp. 1913-1918, 2014.

[61] J. P. Mevy, J. M. Bessiere, J. Rabier et al., "Composition and antimicrobial activities of the essential oil ofTriumfetta rhomboidea Jacq," Flavour and Fragrance Journal, vol. 21, no. 1, pp. 80-83, 2006.

[62] F. Z. Küçükbay, E. Kuyumcu, T. Bilenler, and B Yıldız, "Chemical composition and antimicrobial activity of essential oil of Achillea cretica L. (Asteraceae) from Turkey," Natural Product Research, vol. 26, no. 18, pp. 1668-1675, 2012.

[63] R. Scandiffio, F. Geddo, E. Cottone et al., "Protective effects of (E)- $\beta$-Caryophyllene (BCP) in chronic inflammation," $\mathrm{Nu}$ trients, vol. 12, no. 11, 2020.

[64] S. Koyama, A. Purk, M. Kaur et al., "Beta-caryophyllene enhances wound healing through multiple routes," PloS one, vol. 14, no. 12, Article ID e0216104, 2019. 
[65] M. M. Bashir, M. R. Sharma, and V. P. Werth, "TNF- $\alpha$ production in the skin," Archives of Dermatological Research, vol. 301, no. 1, pp. 87-91, 2009.

[66] J.-F. Rossi, Z.-Y. Lu, M. Jourdan, and B. Klein, "Interleukin-6 as a therapeutic target," Clinical Cancer Research, vol. 21, no. 6, pp. 1248-1257, 2015.

[67] J.-F. Xue, Z.-M. Shi, J. Zou, and X.-L. Li, "Inhibition of PI3K/ AKT/mTOR signaling pathway promotes autophagy of articular chondrocytes and attenuates inflammatory response in rats with osteoarthritis," Biomedicine and Pharmacotherapy, vol. 89, pp. 1252-1261, 2017.

[68] Z. Ruan, S. Wang, W. Yu, and F. Deng, "LncRNA MALAT1 aggravates inflammation response through regulating PTGS2 by targeting miR-26b in myocardial ischemia-reperfusion injury," International Journal of Cardiology, vol. 288, p. 122, 2019.

[69] H. Wu, H. Zhu, Y. Zhuang et al., "LncRNA ACART protects cardiomyocytes from apoptosis by activating PPAR- $\gamma / \mathrm{Bcl}-2$ pathway," Journal of Cellular and Molecular Medicine, vol. 24, no. 1, pp. 737-746, 2020.

[70] Y. Wang, L. Jiang, C. Zhang, Y. Sun, and Q. Tu, "The role of apoptosis-related proteins Bcl-2 and Bax in early pressure ulcer tissues," Chinese Journal of Burns, no. 3, pp. 197-199, 2011.

[71] H. Wang, L. Wang, F. Liang et al., "Exploration on protection mechanism of electroacupuncture with primary secondary point association on rats with chronic myocardial ischemia based on PI3K/AKT signaling pathway," China Journal of Traditional Chinese Medicine and Pharmacy, no. 9, pp. 3475-3480, 2016.

[72] C. Han and Z. Sun, "Effect of moxibustion of expression of eNos protein in skin issue of ratswith PI3K/Akt signaling pathway with pressure ulcer," Journal of Clinical Acupuncture and Moxibustion, vol. 35, no. 7, pp. 57-61, 2019.

[73] E. Bullkich, E. Kimmel, and S. Golan, "A novel ischemia reperfusion injury hereditary tissue model for pressure ulcers progression," Biomechanics and Modeling in Mechanobiology, vol. 18, no. 6, pp. 1847-1866, 2019.

[74] L. Chou, Experimental Study on the Effect of Electroacupuncture on the Repair of Pressure Ulcer Injury and MAPK/ ERK Signaling Pathway in Rats, Heilongjiang University of Chinese Medicine, Harbin, China, 2016.

[75] C. Li, R. Tian, Y. Li, and S. Zhong, "Carapax et plastrum testudinis extracts promote healing of pressure ulcer through regulating Wnt/BMP signaling pathway in hair follicle stem cells," Journal of Guangzhou University of Traditional Chinese Medicine, vol. 36, no. 1, pp. 87-93, 2019.

[76] Z. Yu, T. Li, and J. Wang, "Effects of gold pheasant herb extractive on promoting healing of stage III pressure ulcer in rats via Wnt/ $\beta$-catenin signaling pathway," Journal of Emergency in Traditional Chinese Medicine, vol. 30, no. 4, pp. 612-616, 2021.

[77] J. Li, A. Fu, and L. Zhang, "An overview of scoring functions used for protein-ligand interactions in molecular docking," Interdisciplinary Sciences: Computational Life Sciences, vol. 11, no. 2, pp. 320-328, 2019.

[78] M. Santana de Oliveira, J. N. da Cruz, and W. Almeida da Costa, "Chemical composition, antimicrobial properties of siparuna guianensis essential oil and a molecular docking and dynamics molecular study of its major chemical constituent," Molecules, vol. 25, no. 17, 2020.

[79] B. Li, Study on Anti-inflammatory Activity of Artemisia Argyi Oil, Shanxi Agricultural University, Taiyuan, China, 2013.
[80] Y.-b. Ge, Z.-g. Wang, Y. Xiong, X.-j. Huang, Z.-n. Mei, and Z.-g. Hong, "Anti-inflammatory and blood stasis activities of essential oil extracted from Artemisia argyi leaf in animals," Journal of Natural Medicines, vol. 70, no. 3, pp. 531-538, 2016.

[81] H. Zhou, F. Li, J. Y. Niu et al., "Ferroptosis was involved in the oleic acid-induced acute lung injury in mice," Sheng Li Xue Bao: Acta Physiologica Sinica, vol. 71, no. 5, pp. 689-697, 2019.

[82] B. M. Oh, S. Lee, G. L. Park et al., "Erastin inhibits septic shock and inflammatory gene expression via suppression of the NF«B Pathway," Journal of Clinical Medicine, vol. 8, no. 12, 2019.

[83] Y. Zhou, H. Zhou, L. Hua et al., "Verification of ferroptosis and pyroptosis and identification of PTGS2 as the hub gene in human coronary artery atherosclerosis," Free Radical Biology and Medicine, vol. 171, pp. 55-68, 2021. 